\title{
COMMISSION 24: PHOTOGRAPHIC ASTROMETRY (ASTROMÉTRIE PHOTOGRAPHIQUE)
}

\author{
Report of Meetings, 24 and 29 August 1973
}

President: S. Vasilevskis.

SeCRetary: C. A. Murray.

After welcoming members to the meetings of the Commission, the President reported on changes in the list of members. Six members, Cox, Günther, König, Semirot, Stearns and Wilkens, had died, eight had resigned and 17 new members had been admitted. The current membership was now 57.

The President announced that the following had been nominated as officers and Organizing Committee for the ensuing three years, for approval by the General Assembly:

President: P. Lacroute.

Vice-President: C. A. Murray.

Organizing Committee: A. N. Deutsch, W. Dieckvoss, K. Aa. Strand, H. W. Wood, A. Blaauw, W. Gliese, W van Altena

The President then said that the Executive Committee of the Union had asked Commission Presidents to review the scientific objects and the rules of conduct of their Commissions, and to indicate broad scientific priorities for future work within their particular fields. In the discussion it was agreed that the Commission did not need a set of rigid rules, but that nominations for membership should be made solely by members of the Commission on the basis of the individual candidate's contribution to parallax and proper motion work. The new name of the Commission did not give due weight to modern developments in photo-electric and space techniques for astrometric work, but nevertheless it was generally felt that it would be unwise to seek to change the name of the Commission again. The objectives of the Commission remained the measurement of stellar parallaxes and proper motions by any suitable techniques.

The Executive Committee had also called for views on the publication of Reports on Astronomy in Volume A of the Transactions of the Union. In discussion it was generally agreed that the volume as a whole, by bringing together reports on different branches of the subject, provided a very useful reference on the state of astronomy, and should be continued in its present form.

The President then referred to the draft report of the Working Group on Nomenclature of Faint Stars which had been set up at the Brighton meeting under the Chairmanship of Murray.

The Working Group had proposed a scheme based on rectangular co-cordinates on Palomar Sky Survey prints for the northern hemisphere, with extension to the south when the current surveys had been completed and published. The report had been circulated to all members, and had received a majority approval in a postal ballot. However, there had been two objections and counterproposals, by Thomas and by Giclas and the President regretted that he had acted incorrectly in putting the original draft report to the vote. Accordingly he proposed that the Working Group continue to act, in consultation with the Stellar Data Centre at Strasbourg, to see whether a practicable scheme could be implemented. In discussion, Luyten urged that whenever possible, established identifications such as BD or CoD numbers should continue to be used; editors of journals should insist on precise statements from authors on their sources of nomenclature for stars. Murray remarked that the southern sky charts, to be based on surveys with the ESO and U. K. Schmidt telescopes would have position fiducial marks printed on them to facilitate identifications by means of rectangular co-ordinates, as proposed by the Working Group.

Gliese opened the first scientific session with a review of the need for trigonometric parallaxes in the southern hemisphere. While $45 \%$ of the stars in the two Jenkins Catalogues had negative dec- 
linations, the distribution in declination of stars with the most accurate parallaxes (p.e. $\leqslant 0.006$ ) was:

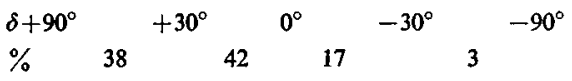

This emphasized the great disparity between the two hemispheres. Since 1963 only the 24 in. refractor at the Cape had contributed to parallaxes in the south. Urgent problems requiring parallax observations in the southern hemisphere included the frequency of nearby M-dwarfs, particularly in the region of the south galactic pole. He welcomed the plans for measurement of parallaxes in this region announced by Murray and Hoffleit at the recent Perth Symposium.

Stock said that he was anxious to undertake astrometric programmes at the new observatory in Venezuela (latitude $+8^{\circ} 48^{\prime}$ ). His equipment would include a $1 \mathrm{~m} \mathrm{f/3} \mathrm{Schmidt,} \mathrm{with} \mathrm{objective}$ prism, a $1 \mathrm{~m} \mathrm{f} / 20$ coudé reflector, a long focus refractor with aperture $65 \mathrm{~cm}$ and focal length $10 \mathrm{~m}$ and a double astrograph with aperture $51 \mathrm{~cm}$ and focal length $3.75 \mathrm{~m}$.

Stoy then spoke about the present status and future needs of proper motions in the southern hemisphere. He drew attention particularly to the large amount of material at present available in published catalogues extending from the early work of Gill, the Carte-du-Ciel catalogues and the AGK1, to the extensive photographic surveys by Yale and the Cape observatories, all of which should be combined into a general catalogue; this could then be used with the modern Gape and Sydney surveys, which will be reduced to the FK4 system by means of the extensive SRS programme, to give a much improved system of fundamental proper motions in the south.

In the second scientific session, van Altena described the various methods of reducing relative trigonometric parallaxes to an absolute system. Current trends in parallax work were toward higher internal accuracy and a reduction in the size of systematic differences between observatories; for example the difference between the system of the new Yerkes parallaxes and that of the 61-in. USNO reflector was only $0 " 0006$ with a standard error of $\pm 0 \% 0025$. He described a new method of reducing parallaxes to absolute, which was based on a revised kinematic model for secular parallaxes, but gave, some weight to the relative proper motion distribution of the reference stars in each particular field.

Murray then reviewed the various methods of deriving absolute proper motions. He pointed out examples of apparent inconsistencies of about 0\%01 p.a. between AGK3 and FK4, and AGK3 and the kinematic reduction method, and suggested that an extensive comparison between AGK3 and the FK4 system, through the GC, should be carried out. He also emphasized the apparent discrepancy between the Lick and Pulkovo results for the rotation in right ascension, but pointed out that systematic errors of a few microns in either or both surveys could account for it; it was an urgent matter to resolve the discrepancy. Dieckvoss warned that the zero point of AGK3 motions in a limited field would have a mean error of about 0.003 ; also, a detailed comparison between AGK3 and GC was being carried out at Bergedorf by de Vegt.

Luyten summarised IAU Symposium No. 54 on 'Problems of Calibration of Absolute Magnitudes and Temperatures of Stars' which was held at Geneva on 1972, September 12-15, and Hoffleit gave a short account of the recent Symposium No. 61 on 'New Problems in Astrometry' which was held in Perth immediately prior to the General Assembly.

After general discussion the following statement of priorities was approved.

Astrometry provides data essential for the solution of such basic problems as the determination of the scale of the Universe, and thus for studies of the luminosities, distribution and kinematics of celestial objects; experience has shown that useful astrometric data can only be acquired by long, sustained series of observations. Commission 24 therefore sets as its most important priorities:

(1) The vigorous continuation of existing trigonometric parallax programmes in the northern hemisphere and a major expansion in the southern hemisphere, with an extension to faint magnitudes over the whole sky.

(2) Consolidation of the zone catalogues over the whole sky into a definitive general catalogue of positions and proper motions relative to the fundamental system. 
(3) Determination of proper motions relative to extragalactic objects over the whole sphere in order to relate the fundamental system to an inertial frame and to provide absolute data for the study of kinematics in the Galaxy.

The following resolutions were proposed and carried unanimously; a preliminary text of Resolution No. 1 had previously been endorsed by a joint meeting of Commissions $8,24,33$ and 40 which was held on 25 August.

1. Commission 24, taking note of Resolutions No. 1 and 2 of IAU Symposium No. 61, emphasises

(a) the extreme importance to fundamental and stellar astronomy of the Yale-Columbia programme for the determination of proper motions relative to extra-galactic objects in the southern hemisphere; it therefore urges that the full programme of first epoch photography in two colours should be completed as soon as possible, and arrangements made to ensure that second epoch photography should start within the next five years.

(b) the potential value of new optical, radio and space techniques for astrometry, and supports programmes for the measurement of proper motions and parallaxes which may be devised for the Large Space Telescope and other space projects.

2. Commission 24 urges that regular and continuing allocation of observing time on suitable large telescopes be made for programmes of trigonometric parallaxes and proper motions of faint stars.

3. Commission 24 , noting the extremely favourable position close to the Equator of the new observatory in Venezuela and the urgent need for basic astrometric data in the southern sky, recommends as highly desirable fields of work

(a) trigonometric parallax observations, especially of stars in the southern hemisphere

(b) positional observations in the equatorial zone between declinations $+30^{\circ}$ and $-30^{\circ}$, with specially high priority for the portion between $0^{\circ}$ and $-30^{\circ}$.

4. Commission 24 , noting the key role played by astrometric data in the solution of many of the most important problems of astrophysics, urges that the methods and achievements of astrometry should be regarded as an integral part of the education of young astronomers.

5. Commission 24 notes with admiration the speed, accuracy and adaptability of the automatic plate scanning and measuring machine developed for the Palomar Schmidt Proper Motion Survey and its great potential for other astronomical problems. It therefore urges that this machine be kept in commission and made available to qualified investigators after the termination of the present project. 